\title{
Radionuclide synovectomy/synoviorthesis (RS) in persons with bleeding disorders: A review of impact of national guidance on frequency of RS using the ATHNdataset
}

Radionuclide synovectomy/synoviorthesis (RS) is a procedure in which radio-active pharmaceuticals are injected into the joint space of someone affected by a proliferative synovial disorder to decrease synovitis. Radiation is delivered to the synovium with minimal penetration to deeper joint structures. Historically, this was a fairly common procedure for persons with haemophilia and other bleeding disorders because it can be performed in an outpatient setting with relatively minimal clotting factor concentrate replacement. ${ }^{1}$ Numerous patients with bleeding disorders have had RS with various isotopes, since this procedure was first described in 1963 with Phosphocol P32 (chromic phosphate P32 suspension), the most commonly utilized isotope in the US. ${ }^{1-8}$ However, patient safety concerns arose after two reports of acute leukaemia in children with haemophilia within 1 year of RS were published in 2002 and 2005, 9,10 which prompted the medical and scientific advisory council (MASAC) to the National Hemophilia Foundation to issue recommendation \# 176 on 15th October $2006 .{ }^{11}$ This recommendation suggested careful consideration of the risk benefit ratio of RS and utilization of a standardized RS protocol. On 18th September 2008 the Food and Drug Administration (FDA) issued a MedWatch regarding the use of P32 for intra-articular use indicating that P32 may increase the risk for leukaemia in certain situations and stating that it was not indicated in the intraarticular treatment of hemarthrosis. ${ }^{12}$ Subsequently in 2012 a large Canadian retrospective cohort study of cancer incidence after RS did not identify a correlation between RS and cancer. ${ }^{13}$ Finally, the first case of solid tumour-Ewing sarcoma diagnosed after RS was published in $2013 .^{8}$ With conflicting publications/recommendations about this procedure, we hypothesized that the American Thrombosis and Hemostasis Network (ATHN) ATHNdataset, which includes US individuals with bleeding disorders who are cared for in Health Resources and Services Administration (HRSA) haemophilia regions (Figure 1 ) and have authorized the sharing of their demographical and clinical information for research would allow interrogation of rates of RS utilization related to selected publications/ guidance documents.

American Thrombosis and Hemostasis Network 3 is a working group of adult and paediatric haematologists and ATHN support staff with interest in RS. The ATHN3 working group designed and performed a retrospective multi-institution, observational cohort study utilizing the data set that included any patient with a bleeding disorder who had undergone a RS procedure through 31/12/2014.
The ATHNdataset includes data contributed from 135 HTC's from eight regions. A standard data collection template was utilized by participating Hemophilia Treatment Centers (HTC's) to validate RS patient data in the ATHNdataset. The chi-square test was utilized to verify the hypothesis. The prevalence of RS in relation to six publications/guidance documents that were determined by the ATHN3 working group was considered. These included publications regarding: two cases of leukaemia the first in 2002 and the second in 2005, the MASAC guideline on RS \#176 in 2006, FDA P32 MedWatch warning regarding P32 in 2008, a comprehensive safety review of Canadian P32 cases in 2012 and a case of Ewing sarcoma in 2013.

As of $31 / 12 / 2014$, the ATHN data set included a total of 19,735 bleeding disorder patients. Of the $135 \mathrm{HTCs}$ contributing data to the ATHNdataset, 38 had reported 362 RS procedures in 196 patients. Age at RS ranged from 4 to 72 years (median 15 years). Ninety-five per cent of patients who had RS had haemophilia A or B and $97 \%$ were male. Overall, the proportions of patients of different races and ethnicity who had RS did not differ from data set participants who did not have RS

The mean annual number of procedures increased between leukaemia case \#1 and leukaemia case \#2 from 18 to 21 (Figure 2). The mean number of annual procedures decreased slightly after MASAC guidance in 2006 and has continued to decline thereafter. Mean RS instillations decreased from 13 per year before the MedWatch warning in 2008 to 12 in 2012, and to 7 by 31/12/2014. A total of 149 (43\%) of injections were performed between the first leukaemia report and the Canadian safety report in 2012. The annualized number of RS injections performed after the Canadian safety report in 2012 was 7 and the total number of RS procedures continued to decline after the Ewing sarcoma publication in 2013 to 3 in the year 2014.

There were significantly fewer patients who had repeated RS after the MASAC guidelines ( $17 \%$ vs. $83 \%$; $P<.001)$.We did not find race or ethnicity related differences in RS rates after the first publication of MASAC in 2006. About $27 \%$ of Caucasian and $25 \%$ of Non-Caucasian patients had RS after the MASAC guidelines $(P=.821)$. The percentage of procedures performed in Hispanic or non-Hispanic patients after MASAC guidelines did not differ too much, $32 \%$ vs. $25 \%(P=.480)$. MASAC guidance did appear to impact the use of RS in some regions but not others $(P<.001)$. After the MASAC guidelines the number of RS procedures dropped substantially for New England (from 31 to 3), Great Lakes (from 90 to 16), Mountain States (from 16 to 2), 


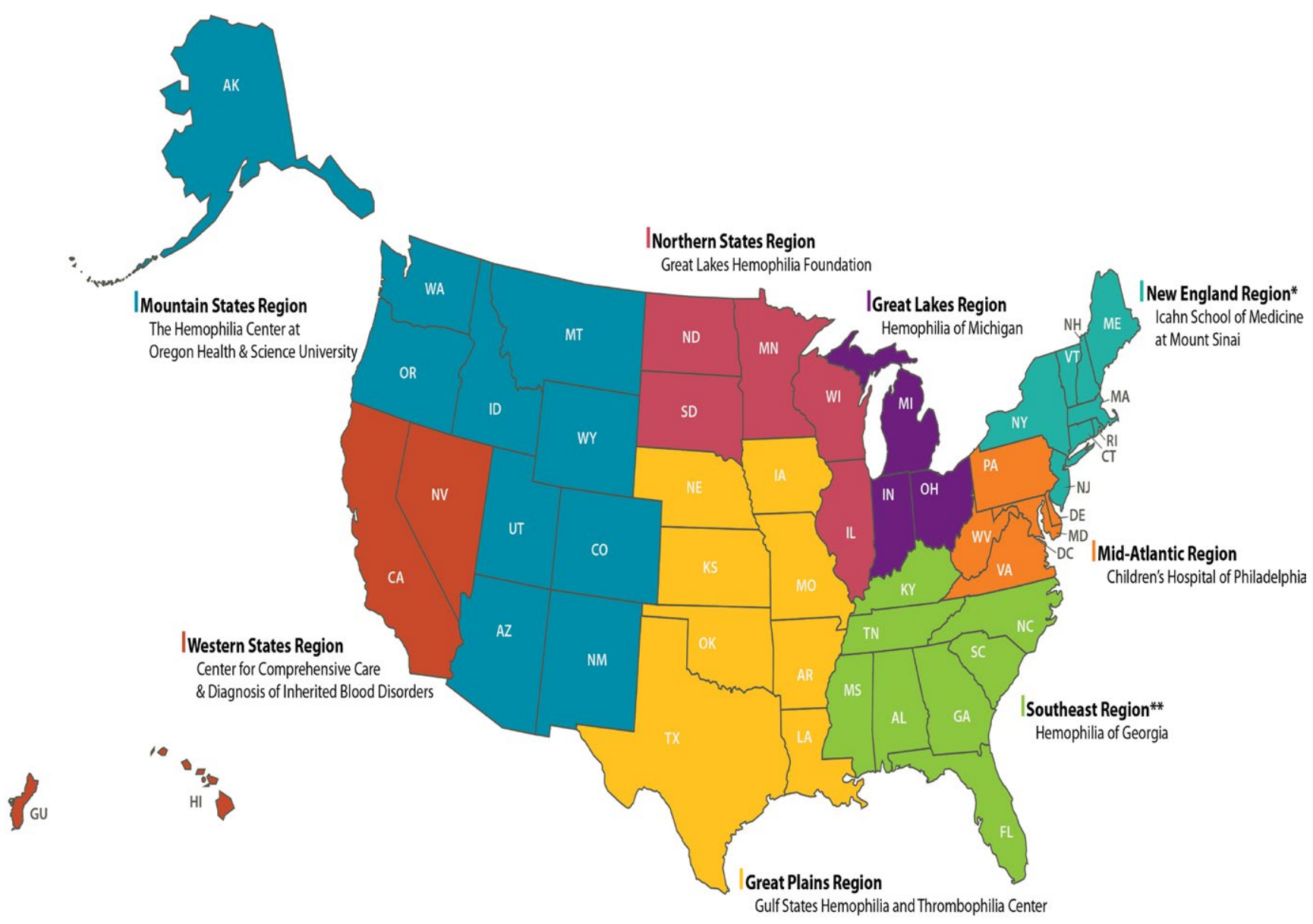

"For CDC grants and activities, New England Region functions as Region I (Regional Center: New England Medical Center, University of Massachusetts, Worcester, MA. States include: CT, MA, ME, NH, RI, VT) and Region II (Regional Center: Ichan School of Medicine At Mount Sinai, New York, NY. States include: NJ, NY, PR)

*For CDC grants and activities, Southeast Region functions as Region IV-N (Regional Center: University of North Carolina at Chapel Hill, Chapel Hill, NC. States include: KY, NC, TN, SC) and Region IV-S

(Regional Center: Hemophilia of Georgia, Atlanta, GA. States include: AL, FL, GA, MS)

FIG URE 1 US Hemophilia Treatment Center (HTC) Network Regions and Regional Centers Included in the American Thrombosis and Hemostasis Network 3 (ATHN) Review. (reproduced with permission from ATHN)

Great Plains (from 19 to 6) and the Southeast (from 80 to 38) regions. Northern States (from 8 to 6) and Western States (from 17 to 14) did not see the substantial reduction in the amount of RS procedures as the other regions. Interestingly enough, there was no significant difference between number of RS procedures performed in patients with inhibitors (32.8\%) compared to patients without inhibitors (29.5\%) after MASAC guidelines $(P=.658)$.

Our study was limited by its retrospective database design. Practice changes such as increasing use of primary and secondary prophylaxis with resultant decrease in arthropathy as well as difficulty in obtaining P32 after the MedWatch warning may have influenced RS rates and could not be independently evaluated. A potential study of the rates of surgical synovectomies over this period may help discern these variables. In our study, we were unable to evaluate this because some patients who underwent RS also had surgical synovectomies, and these variables were not mutually exclusive. Additionally, the short supply of P32 may have been a confounder of the impact of MASAC guidelines on the regional change in procedure as well.
The data suggests that the MASAC guidelines may influence general practice. Another interesting observation was that the MASAC guidelines seem to be more impactful in certain regions as opposed to others. Regional differences may have been descriptive of individual preferences of groups of practitioners or institutions driven by factors including availability of someone to handle the radioactive isotope which is highly regulated, limited availability of experts to administer the injections and size of centres and patient volumes justifying purchase of the isotope. Additionally, the ATHNdataset was utilized as a high quality data set in this study which paves the way for future studies using the ATHNdataset that may require evaluating a large population of patients with bleeding disorders in multiple centres.

\section{ACKNOWLEDGEMENTS}

The ATHNdataset, a HIPAA compliant limited data set under the stewardship of the ATHN, was developed through collaboration with over 135 ATHN-affiliated Haemophilia Treatment Centers across the US. 


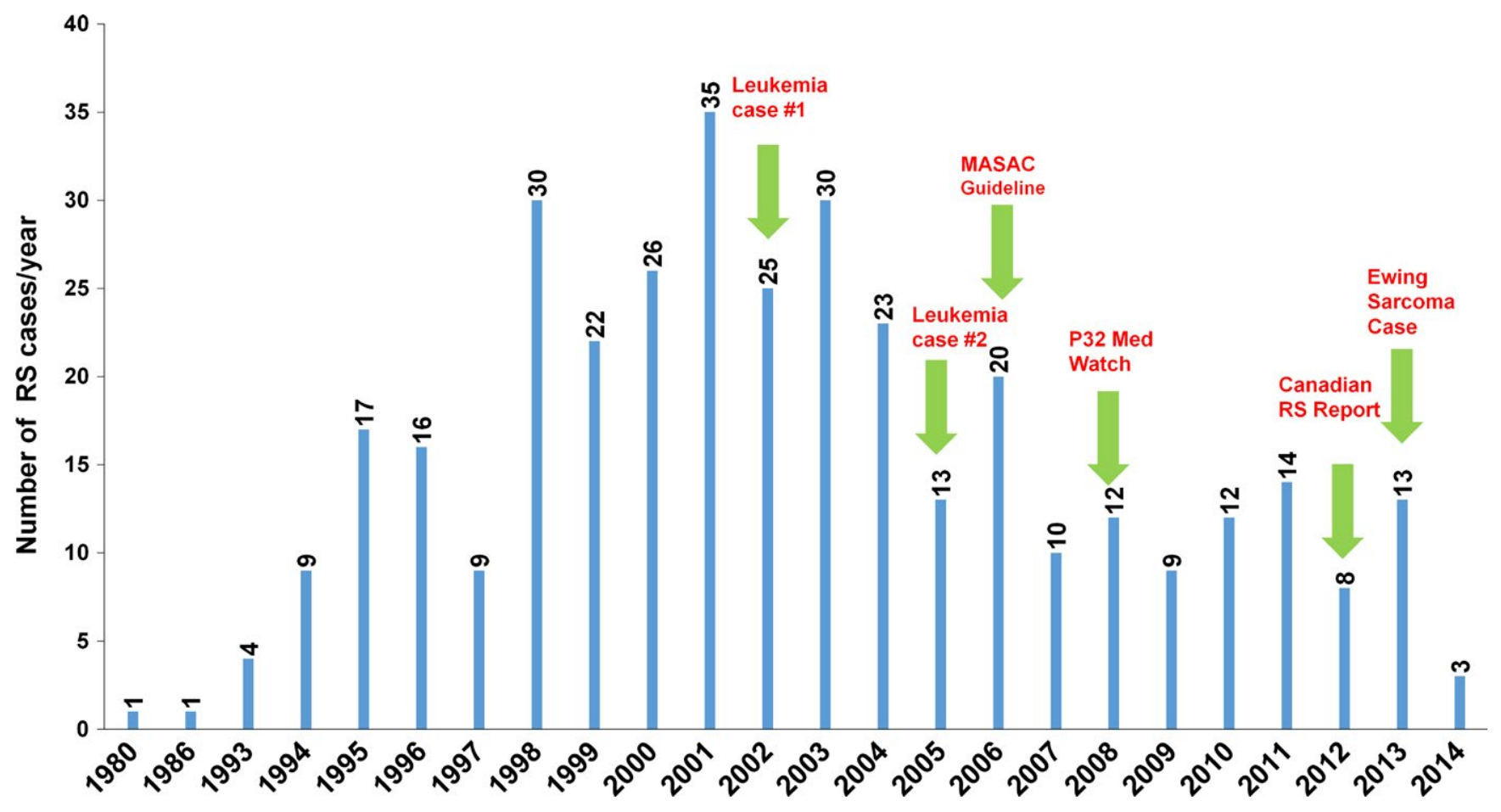

FIGURE 2 Distribution of radioactive synovectomy (RS) Procedures Over Different years as per the American Thrombosis and Hemostasis Network dataset. MASAC: Medication and Scientific Advisory Council

\section{DISCLOSURES}

RS, DS, DC, CK, GY, ML, AS, RG, SA and AS have no competing interests to declare. AD has acted as a consultant to Bayer, Baxalta/ Shire, CSL Behring and Medscape and has received research support from Bayer, Baxalta/Shire, CSL Behring, Octapharma, Kedrion and Pfizer. APW has acted as paid consultant for NovoNordisk, Shire and Bayer. CEM has acted as a paid consultant to Baxalta/ Shore and receives research funding as an investigator for Baxalta/ Shire, Biogen and Roche/Genentech. JD serves as a member of the medical advisory board for Novo Nordisc, Bayer Pharmaceuticals, CSL Behring, Baxalta/Shire, Biogen Idec, Grifols and is paid a fee for speaking for Excel CME. MT is a consultant on the advisory board for NovoNordisk, Baxalta, Grifols, Pfizer and Biogen. He is on speaker bureau for Grifols and Biogen and is grant reviewer for Pfizer. He has received research grant from Grifols, he has an investigator initiated study with NovoNordisk and is principal investigator for programme grant for HRSA and CDC. RS has acted as a paid consultant to Biogen, Pfizer, Octapharma, LFB, Hema Biologics, Shire and Emergent Solutions. SC has been a consultant for NovoNordisk, Hema Biologics and has received grant funding from Pfizer. SP serves on the board of directors for ATHN.

\section{AUTHOR CONTRIBUTIONS}

$A D$ designed the research study and critically revised the manuscript. RS performed the research, analysed the data and wrote the manuscript. DC conducted the statistical analysis and data interpretation. All ATHN investigators made substantial contributions to research design, conducted the research study and contributed research subjects. All authors approved the final and submitted versions of the manuscript.

R. Sharma ${ }^{1}$ iD

A. Dunn ${ }^{2}$

D. Aschman ${ }^{3}$

D. Cheng ${ }^{3}$

A. Wheeler ${ }^{4}$

A. Soni ${ }^{5}$

C. McGuinn ${ }^{6}$

C. Knoll ${ }^{7}$

D. T. Stein ${ }^{8}$

G. Young ${ }^{9}$ iD

J. French ${ }^{10}$

J. Sanders ${ }^{11}$

J. A. Davis ${ }^{12}$

M. Tarantino ${ }^{13}$

M. Lim ${ }^{14}$ iD

R. Gruppo ${ }^{15}$

R. Sidonio ${ }^{16}$

S. Ahuja ${ }^{17}$

S. Carpenter ${ }^{18}$

S. Pipe ${ }^{19}$

A. Shapiro ${ }^{20}$ 
${ }^{1}$ BloodCenter of Wisconsin, Milwaukee, WI, USA

${ }^{2}$ Nationwide Children's Hospital, Columbus, OH, USA

${ }^{3}$ American Thrombosis and Hemostasis Network, Riverwoods, IL, USA

${ }^{4}$ Vanderbilt University Medical Center, Nashville, TN, USA

${ }^{5}$ Center for Comprehensive Care and Diagnosis of Inherited Blood

Disorders, Orange, CA, USA

${ }^{6}$ Weill Cornell Medicine, New York, NY, USA

${ }^{7}$ Arizona Hemophilia and Thrombosis Center, University of Arizona Health

Sciences Center, Tucson, AZ, USA

${ }^{8}$ Northwest Ohio Hemophilia Treatment Center, Toledo, OH, USA

${ }^{9}$ Childrens Hospital Los Angeles, Los Angeles, CA, USA

${ }^{10}$ Palmetto Health Richland, Columbia, SC, USA

${ }^{11}$ Cook Children's Hospital, Fort Worth Bleeding Disorders Program, Fort

Worth, TX, USA

${ }^{12}$ University of Miami Comprehensive Pediatric Hemophilia Treatment

Center, Miami, FL, USA

${ }^{13}$ Bleeding and Clotting Disorders Institute, University of Illinois College of Medicine-Peoria, Peoria, IL, USA

${ }^{14}$ UNC Comprehensive Hemophilia Diagnostic and Treatment Center, Chapel Hill, NC, USA

${ }^{15}$ Cincinnati Children's Hospital Medical Center, Cincinnati, OH, USA

${ }^{16}$ Emory/Children's Healthcare of Atlanta Comprehensive Hemophilia Program, Atlanta, GA, USA

${ }^{17}$ Rainbow Babies \& Children's Hospital, University Hospitals Case Medical Center, Cleveland, $\mathrm{OH}$, USA

${ }^{18}$ Children's Mercy Hospital, Kansas City, MO, USA

${ }^{19}$ Department of Pediatrics and Pathology, University of Michigan, Ann Arbor, MI, USA

${ }^{20}$ Indiana Hemophilia and Thrombosis Center, Indianapolis, IN, USA

Correspondence

Ruchika Sharma,

BloodCenter of Wisconsin, Milwaukee, WI, USA.

Email: ruchika.sharma@bcw.edu efficacy and recommendation for a standardized treatment protocol. [Review] [104 refs]. Thromb Haemost. 2002;87:383-393.

2. Ansell BMCA, Mallard JR, Bywaters EG. Evaluation of intra-articular colloidal gold Au 198 in the treatment of persistent knee effusions. Ann Rheum Dis. 1963;22:435-439.

3. Heim M, Goshen E, Amit Y, Martinowitz U. Synoviorthesis with radioactive Yttrium in haemophilia: Israel experience. Haemophilia. 2001;7(Suppl 2):36-39.

4. Ahlberg A, Pettersson H. Synoviorthesis with radioactive gold in hemophiliacs. Clinical and radiological follow-up. Acta Orthop Scand. 1979;50:513-517.

5. Merchan EC, Magallon M, Martin-Villar J, Galindo E, Ortega F, Pardo JA. Long term follow up of haemophilic arthropathy treated by Au-198 radiation synovectomy. Int Orthop. 1993;17: 120-124.

6. Rivard GE, Girard M, Belanger R, Jutras M, Guay JP, Marton D. Synoviorthesis with colloidal 32P chromic phosphate for the treatment of hemophilic arthropathy. [see comment]. J Bone Joint Sur American Volume. 1994; 76:482-488.

7. Rodriguez-Merchan EC, Quintana M, De la Corte-Rodriguez H, Coya J. Radioactive synoviorthesis for the treatment of haemophilic synovitis. Haemophilia. 2007;13(Suppl 3):32-37.

8. Thomas S, Mendes JD, Souza SA, Lorenzato CS, Assi PE, Pacheco LRL, et al. Radioactive synovectomy with 90yttrium and 153samarium hydroxyapatite in haemophilic joints: preliminary study on radiation safety. Haemophilia. 2013;19:632-636.

9. Manco-Johnson MJ, Nuss R, Lear J, Wiedel J, Geraghty SJ, Hacker MR, et al. 32P Radiosynoviorthesis in children with hemophilia. J Pediatr Hematol Oncol. 2002;24:534-539.

10. Dunn AL, Manco-Johnson M, Busch MT, Balark KL, Abshire TC. Leukemia and P32 radionuclide synovectomy for hemophilic arthropathy. J Thromb Haemost. 2005;3:1541-1542.

11. Council NHFMaSA. MASAC recommendations regarding radionucleotide synovectomy. MASAC Document \#194 replaces \#176: National Hemophilia Foundation. Available at: https://www.hemophilia.org/ sites/default/files/document/files/176.pdf. [Accessed June 26, 2010].

12. Administration USFD. Phosphocol P 32 (Chromic Phosphate P 32 Suspension). Safety alerts for human medical products. [Accessed October 25, 2016].

13. Infante-Rivard C, Rivard GE, Derome F, Cusson A, Winikoff R, Chartrand $\mathrm{R}$, et al. A retrospective cohort study of cancer incidence among patients treated with radiosynoviorthesis. Haemophilia. 2012;18:805-809.

\section{REFERENCES}

1. Dunn AL, Busch MT, Wyly JB, Abshire TC. Radionuclide synovectomy for hemophilic arthropathy: a comprehensive review of safety and 